\title{
Correction: Glucocorticoid treatment for non-cerebral diseases in children and adolescents is associated with differences in uncinated fasciculus microstructure
}

Martin Vestergaard, William F. C. Baaré, Sara K. Holm, Camilla G. Madsen, Olaf B. Paulson (D, Alfred P. Born, Peter Uldall, Hartwig R. Siebner and Kathrine Skak Madsen

(c) The Author(s), under exclusive licence to the International Pediatric Research Foundation, Inc 2021

Pediatric Research (2022) 91:1008; https://doi.org/10.1038/s41390-021-01736-8

Correction to: Pediatric Research https://doi.org/10.1038/s41390-

021-01394-w, published online 31 March 2021

In this article the wrong figure appeared as Fig. 3; the figure should have appeared as shown below. The original article has been corrected.

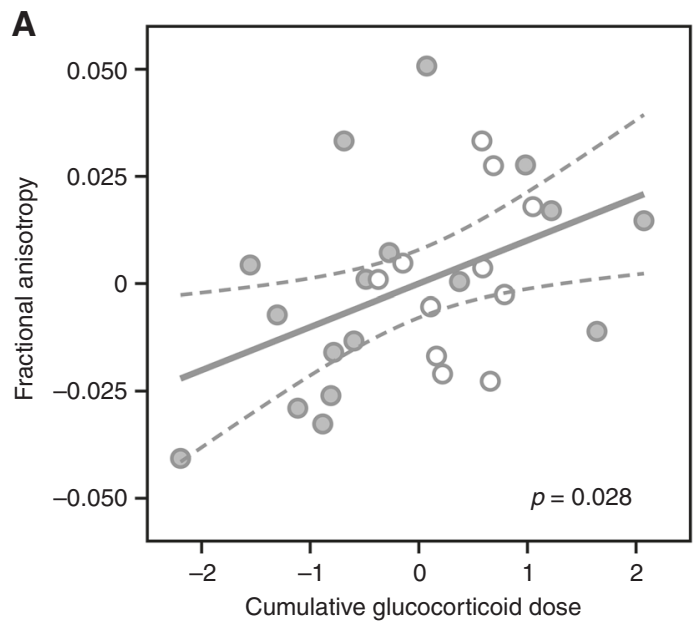

Nephrotic patients

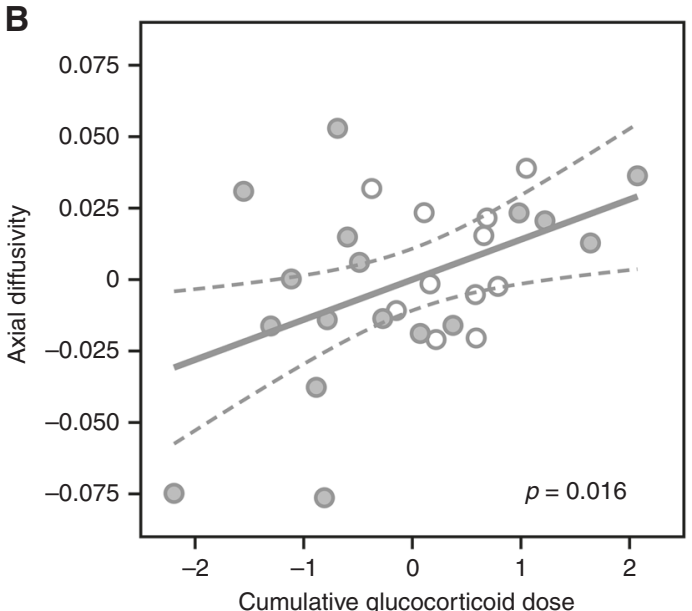

Rheumatic patients 